\title{
ON THE MONODROMY AT ISOLATED SINGULARITIES OF WEIGHTED HOMOGENEOUS POLYNOMIALS
}

BY

BENJAMIN G. COOPER

\begin{abstract}
Assume $f: \mathbf{C}^{\mathbf{m}} \rightarrow \mathbf{C}$ is a weighted homogeneous polynomial with isolated singularity, and define $\phi: S^{2 m-1}-f^{-1}(0) \rightarrow S^{1}$ by $\phi(\vec{z})=f(\vec{z}) /|f(\vec{z})|$. If the monomials of $f$ are algebraically independent, then the closure $F_{0}$ of $\phi^{-1}(1)$ in $S^{2 m-1}$ admits a deformation into the subset $G$ where each monomial of $f$ has nonnegative real values. For the polynomial $f\left(z_{1}, \ldots, z_{m}\right)=z_{1}^{a_{1}} z_{2}+\cdots+z_{m-1}^{a_{m-1}} z_{m}+z_{m}^{a_{m}} z_{1}, G$ is a cell complex of dimension $m-1$, invariant under a characteristic map $h$ of the fibration $\phi$, and the inclusion $G \rightarrow \bar{F}_{0}$ induces isomorphisms in homology. To compute the homology of the link $K=f^{-1}(0) \cap S^{2 m-1}$ it thus suffices to calculate the action of $h_{*}$ on $H_{m-1}(G)$. Let $d=a_{1} a_{2} \cdots a_{m}+(-1)^{m-1}$. Let $w_{1}, w_{2}, \ldots, w_{m}$ be the weights associated with $f$, satisfying $a_{j} / w_{j}+1 / w_{j+1}=1$ for $j=$ $1,2, \ldots, m-1$ and $a_{m} / w_{m}+1 / w_{1}=1$. Let $n=d / w_{1}, q=\operatorname{gcd}(n, d), r=q+$ $(-1)^{m}$. Then $H_{m-2}(K)=Z^{r} \oplus Z_{d / q}$ and $H_{m-1}(K)=Z^{r}$.
\end{abstract}

The purpose of this paper is to calculate for the complex polynomial $f$ defined by

$$
f\left(z_{1}, z_{2}, \ldots, z_{m}\right)=z_{1}^{a_{1}} z_{2}+z_{2}^{a_{2} z_{3}}+\cdots+z_{m-1}^{a_{m-1}} z_{m}+z_{m}^{a_{m}} z_{1}
$$

the integral homology of the $(2 m-3)$-manifold $K$ defined by $K=\left\{\left(z_{1}, \ldots, z_{m}\right)\right.$ $\in \mathbf{C}^{m}:\left|z_{1}\right|^{2}+\cdots+\left|z_{m}\right|^{2}=1$ and $\left.f\left(z_{1}, \ldots, z_{m}\right)=0\right\}$. This contributes to a project begun by Milnor [3] and continued by Milnor and Orlik [4], Orlik and Wagreich [9], Orlik [6], Orlik and Randell [8], and others, to compute invariants which will help to describe the topology of a hypersurface defined by a complex polynomial near an isolated singularity. The results of this paper can be described briefly. Let $d=a_{1} a_{2} \cdots a_{m}+(-1)^{m-1}$, and let $q$ be the greatest common divisor of $d$ and $a_{2} a_{3} \cdots a_{m}-a_{3} a_{4} \cdots a_{m}+a_{4} \cdots a_{m}-\cdots+(-1)^{m-2} a_{m}+(-1)^{m-1}$. Then $K$ is $(m-3)$-connected, $H_{m-2}(K)$ is the direct sum of a free abelian group of rank $q+(-1)^{m}$ and a cyclic group of order $d / q$, and $H_{m-1}(K)$ is free abelian of rank $q+(-1)^{m}$.

I am grateful to Peter Orlik for the survey article [7] which brought the problem to my attention and which has a good bibliography on this and related subjects.

1. Preliminaries. Let $f: \mathbf{C}^{m} \rightarrow \mathbf{C}$ be a polynomial, and let $V=f^{-1}(0)$. If $V$ has a singularity at $\vec{z}_{0}$, that is, all the partial derivatives of $f$ vanish there, let $S_{\varepsilon}$ denote the $(2 m-1)$-sphere in $\mathbf{C}^{m}$ of radius $\varepsilon$, centered at $\vec{z}_{0}$, and write $K=V \cap S_{e}$. Then for all $\varepsilon$ sufficiently small, the portion of $V$ inside $S_{\varepsilon}$ is homeomorphic to the cone over $K[3$, p. 18], and so we are interested in the topology of $K$.

Received by the editors September 3, 1980 and, in revised form, January 28, 1981.

1980 Mathematics Subject Classification. Primary 14B05; Secondary 32C40.

Key words and phrases. Isolated singularity, weighted homogeneous polynomial, Milnor fibre, monodromy. 
Milnor showed in [3] that the function $\phi: S_{e}-K \rightarrow S^{1}$ defined by $\phi(\vec{z})=$ $f(\vec{z}) /|f(\vec{z})|$ is the projection of a locally trivial fibre space and that if the singularity $\vec{z}_{0}$ is isolated (and $\varepsilon$ is sufficiently small), then each fibre $F_{\theta}=\phi^{-1}\left(e^{i \theta}\right)$ has the homotopy type of a bouquet of $(m-1)$-spheres. The number of spheres involved, called $\mu$, is the multiplicity of $\vec{z}_{0}$ as a zero of the gradient of $f$, a measure of the degeneracy of the critical point. Furthermore, still assuming the singularity is isolated, the closure $\bar{F}_{\theta}$ is a smooth $(2 m-2)$-dimensional manifold with boundary whose interior is $F_{\theta}$ and whose boundary is $K$. The manifold $K$ is $(m-3)$ connected.

Because $S_{\varepsilon}-K$ is fibred over a circle, there exist one-parameter families of homeomorphisms $h_{t}: F_{0} \rightarrow F_{t}$ for $0 \leqslant t \leqslant 2 \pi$, with $h_{0}$ the identity map. Choose such a family and let $h$ denote $h_{2 \pi}$. The map $h$ is called a characteristic map for the fibration, and the induced automorphism $h_{*}: H_{m-1} F_{0} \rightarrow H_{m-1} F_{0}$, which is independent of the choice of family $\left\{h_{t}\right\}$, is called the monodromy of $f$ at the singularity $\vec{z}_{0}$. When $m \geqslant 3$, by using Alexander and Poincaré duality to substitute in the Wang sequence of the fibration, we obtain an exact sequence

$$
0 \rightarrow H_{m-1} K \rightarrow H_{m-1} F_{0} \stackrel{I_{*}-h_{*}}{\rightarrow} H_{m-1} F_{0} \rightarrow H_{m-2} K \rightarrow 0,
$$

where $I$ is the identity map of $F_{0}[3, \mathrm{pp} .67-68]$. Hence in the isolated singularity case, $h_{*}$ determines the homology of $K$.

Let $w_{1}, w_{2}, \ldots, w_{m}$ be positive rational numbers. The polynomial $f\left(z_{1}, z_{2}, \ldots, z_{m}\right)$ is weighted homogeneous with weights $\left(w_{1}, w_{2}, \ldots, w_{m}\right)$ if it can be expressed as a linear combination of monomials $z_{1}^{e_{1}} z_{2}^{e_{2}} \cdots z_{m}^{e_{m}}$ for which $e_{1} / w_{1}+$ $e_{2} / w_{2}+\cdots+e_{m} / w_{m}=1$. For a weighted homogeneous polynomial, the only possible isolated critical point is the origin. Weighted homogeneous polynomials for which the origin is an isolated critical point play an important role in Arnold's theory of normal forms for singularities (see, for example, [1]). Assume from now on that $f$ is such a polynomial.

Then the radius $\varepsilon$ of the sphere $S_{e}$ is immaterial. Specifically, from the evident identity

$$
f\left(z_{1} \cdot \exp \left\{w / w_{1}\right\}, z_{2} \cdot \exp \left\{w / w_{2}\right\}, \ldots, z_{m} \cdot \exp \left\{w / w_{m}\right\}\right)=e^{w} f\left(z_{1}, z_{2}, \ldots, z_{m}\right)
$$

we obtain both an explicit formula $h\left(z_{1}, z_{2}, \ldots, z_{m}\right)=\left(z_{1} \cdot \exp \left\{2 \pi i / w_{1}\right\}, z_{2}\right.$. $\left.\exp \left\{2 \pi i / w_{2}\right\}, \ldots, z_{m} \cdot \exp \left\{2 \pi i / w_{m}\right\}\right)$ for a characteristic map and, for any positive $\varepsilon$ and $\varepsilon^{\prime}$, a diffeomorphism $S_{\varepsilon} \rightarrow S_{\varepsilon^{\prime}}$ which is equivariant with respect to $h$, which maps $K=V \cap S_{\varepsilon}$ to $K^{\prime}=V \cap S_{e^{\prime}}$, and which restricts to a fibre-preserving map $S_{\varepsilon}-K \rightarrow S_{\varepsilon^{\prime}}-K^{\prime}$. (Compare [9, p. 218].) We shall in what follows take $\varepsilon=1$ and take for characteristic map the map $h$ defined just above.

Milnor and Orlik [4, p. 387] showed that the multiplicity $\mu$, which equals the rank of $H_{m-1} F_{0}$, is $\left(w_{1}-1\right)\left(w_{2}-1\right) \cdots\left(w_{m}-1\right)$.

Following [8], let us call a weighted homogeneous polynomial $g\left(z_{1}, \ldots, z_{k}\right)$ with isolated critical point decomposable if, after suitable renumbering of the variables, $g\left(z_{1}, \ldots, z_{k}\right)=g_{1}\left(z_{1}, \ldots, z_{l}\right)+g_{2}\left(z_{l+1}, \ldots, z_{k}\right)$, where $g_{1}$ and $g_{2}$ have isolated critical points at the origins of $\mathbf{C}^{l}$ and $\mathbf{C}^{k-l}$, respectively. Oka showed in [5] that the Milnor fibre for $g$ is then homotopy equivalent to the reduced join of the fibres for 
$g_{1}$ and $g_{2}$. The monodromy of $g$ is therefore the tensor product of the monodromies of $g_{1}$ and $g_{2}$. The polynomial which is the subject of this paper is indecomposable in the sense above, except in the degenerate case $a_{1}=a_{2}=\cdots=a_{m}=1$ (see §).

The main purpose of this paper is thus to compute the kernel and cokernel of $I_{*}-h_{*}: H_{m-1} F_{0} \rightarrow H_{m-1} F_{0}$ for the weighted homogeneous polynomial $f\left(z_{1}, \ldots, z_{m}\right)=z_{1}^{a_{1} z_{2}}+\cdots+z_{m-1}^{a_{m-1}} z_{m}+z_{m}^{a_{m}} z_{1}$, assuming $a_{1} a_{2} \cdots a_{m}>1$. The computation is in two parts. First, the closure $\bar{F}_{0}$ is deformed into a subspace $G$, invariant under the explicit characteristic map $h$ described above. The subspace $G$ is the set where each monomial of $f$ separately has the same argument, i.e., is real and nonnegative. The deformation exists for a whole class of weighted homogeneous polynomials, and its construction occupies $\$ \S 4-14$. In the case at hand, $G$ is a cell complex of dimension $m-1$. To calculate $h_{*}: H_{m-1} G \rightarrow H_{m-1} G$, and hence the homology of $K$, it is useful to filter $G$ by the number of nonzero complex coordinates and study the resulting spectral sequence. The filtration corresponds to a sort of cellular decomposition of $G$ which is described in $\S 15$. The remaining $\S \S 16-22$ contain the actual calculations.

2. First let us dispose of the degenerate case. Suppose $g\left(z_{1}, \ldots, z_{m}\right)=z_{1} z_{2}+$ $z_{2} z_{3}+\cdots+z_{m-1} z_{m}+z_{m} z_{1}$. If $m \equiv 0(\bmod 4)$, then the critical point at the origin is not isolated. In all other cases we can make linear changes of variable to decompose $g$ in the sense above. If $m$ is odd, we may, for example, take $z_{1}^{\prime}=z_{1}+$ $z_{3}, \quad z_{3}^{\prime}=z_{3}+z_{5}, \ldots, z_{m-2}^{\prime}=z_{m-2}+z_{m}, z_{m}^{\prime}=z_{m}, z_{2}^{\prime}=z_{2}+z_{m}, z_{4}^{\prime}=z_{4}-$ $z_{m}, \ldots, z_{m-1}^{\prime}=z_{m-1}+(-1)^{(m-3) / 2} z_{m}$. Then $g\left(z_{1}, z_{2}, \ldots, z_{m}\right)=z_{1}^{\prime} z_{2}^{\prime}+z_{3}^{\prime} z_{4}^{\prime}$ $+\cdots+z_{m-2}^{\prime} z_{m-1}^{\prime}+(-1)^{(m-1) / 2}\left(z_{m}^{\prime}\right)^{2}$. If $m \equiv 2(\bmod 4)$, we may take $z_{1}^{\prime}=z_{1}+$ $z_{3}, \quad z_{3}^{\prime}=z_{3}+z_{5}, \ldots, z_{m-1}^{\prime}=z_{m-1}+z_{1}$, and $z_{2 k}^{\prime}=z_{2 k}$ for all $k$. Then $g\left(z_{1}, z_{2}, \ldots, z_{m}\right)=z_{1}^{\prime} z_{2}^{\prime}+z_{3}^{\prime} z_{4}^{\prime}+\cdots+z_{m-1}^{\prime} z_{m}^{\prime}$. Therefore let us assume from now on that $a_{1} a_{2} \cdots a_{m}>1$.

3. In addition to the continuing assumption that $f\left(z_{1}, z_{2}, \ldots, z_{m}\right)$ is a weighted homogeneous polynomial with isolated critical point, consider from now on the following additional assumption. Write $f$ as a sum $f_{1}+f_{2}+\cdots+f_{r}$ of monomials, say

$$
f_{j}\left(z_{1}, z_{2}, \ldots, z_{m}\right)=c_{j} z_{1}^{e_{1}, z_{2}^{e_{1}, 2}} \cdots z_{m}^{e_{j, m}},
$$

where $c_{j}$ is a complex constant, and assume that the $m$-tuples $\left(e_{j, 1}, e_{j, 2}, \ldots, e_{j, m}\right)$ of integers for $j=1,2, \ldots, r$, considered as vectors in $Q^{m}$, form an independent set. This condition has considerable overlap with the continuing assumption; it implies the existence of weights $\left(w_{1}, w_{2}, \ldots, w_{m}\right)$ with the desired homogeneity property, though not their positivity (nor their uniqueness if the number $r$ of monomials is less than $m$ ). It rules out polynomials such as $f\left(z_{1}, z_{2}\right)=z_{1}^{2}+z_{1} z_{2}+z_{2}^{2}$, but it does apply to the polynomial which is the ultimate subject of this paper.

Define a subset $G$ of the sphere $S^{2 m-1}$ by $G=\left\{\vec{z} \in S^{2 m-1}\right.$ : for each $j, f_{j}(\vec{z})$ is a nonnegative real number $\}$. Note that $G \subseteq \overline{F_{0}}=\left\{\vec{z} \in S^{2 m-1}: f(\vec{z})\right.$ is a nonnegative real number . 


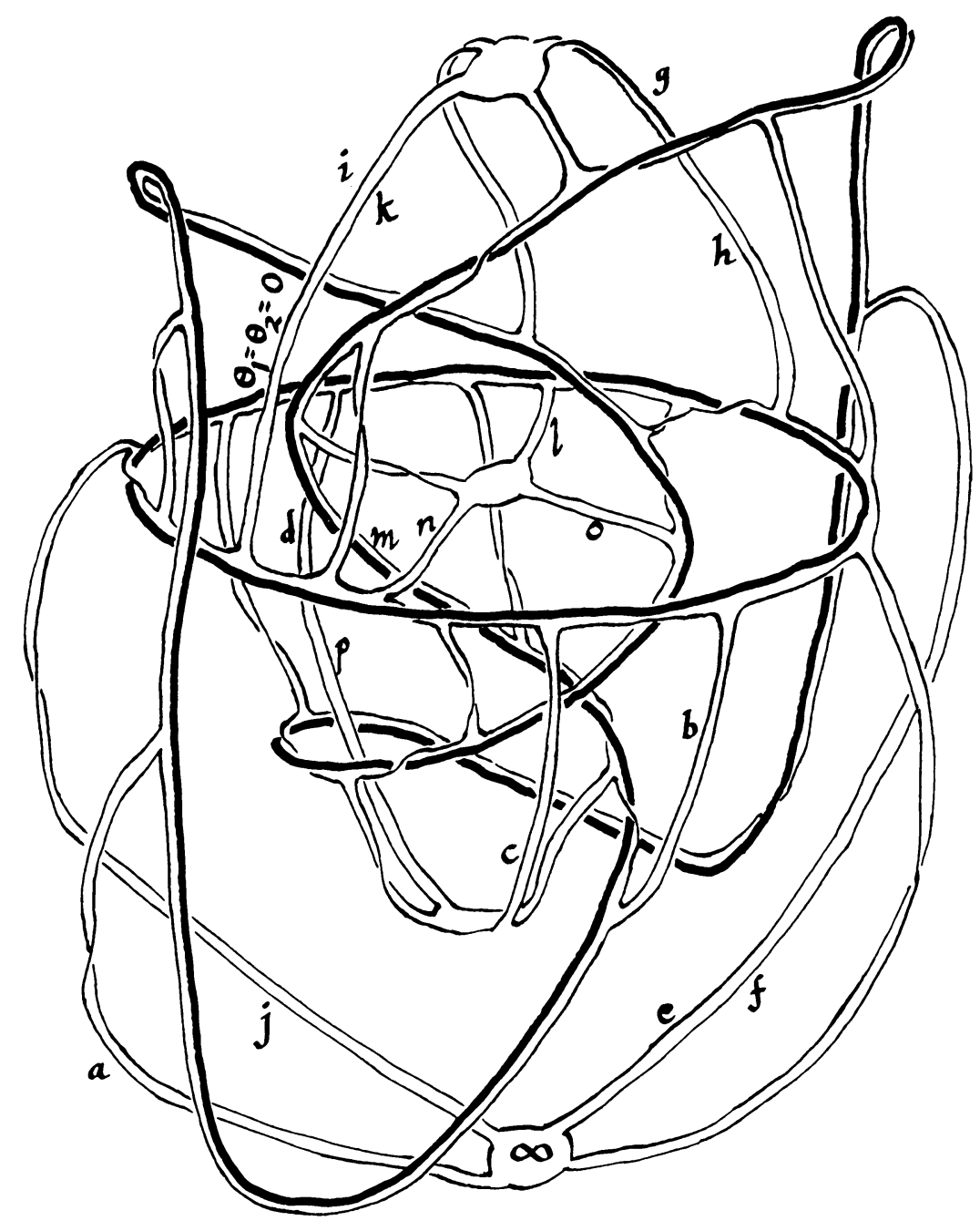

FIGURE 1

It would be useful to be able to assert that $G$ is a deformation retract of $\bar{F}_{0}$. Figures 1 and 2 are psychologically persuasive evidence for this assertion. Figure 1 shows part of the image under the stereographic projection $\left(z_{1}, z_{2}\right) \mapsto$ $\left(1-\operatorname{Im}\left(z_{2}\right)\right)^{-1}\left(z_{1}, \operatorname{Re}\left(z_{2}\right)\right)$ from $S^{3}$ to $\mathbf{R}^{3} \cup\{\infty\}$ of the closure of the fibre $F_{0}$ for the polynomial $f\left(z_{1}, z_{2}\right)=z_{1}^{2} z_{2}+z_{2}^{4}$. The drawing is not to scale and the point at infinity has been moved into view. The boundary $K$ is in heavy outline. Along the center lines of the bands which are shown connecting the circle $\left\{z_{2}=0\right\}$ to either the torus knot $\left\{z_{1}^{2}+z_{2}^{3}=0\right\}$ or one of the four points on the circle $\left\{z_{1}=0\right\}$, the arguments of both $z_{1}$ and $z_{2}$ are constant; if we write $z_{j}=\left|z_{j}\right| \exp \left\{i \theta_{j}\right\}$, then the bands meet the circle $\left\{z_{2}=0\right\}$ at $\theta_{1}=k \pi / 8, k=0,1,2, \ldots, 15$. The surface is to be completed by sewing on sixteen disks, whose boundaries are the curves marked " $a$ ", " $b ", " c ", \ldots$, " $p$ ". Figure 2 shows the position of the torus knot at a late stage in an evident deformation retraction from $\bar{F}_{0}$ to $G$, which is now also in bold outline. 


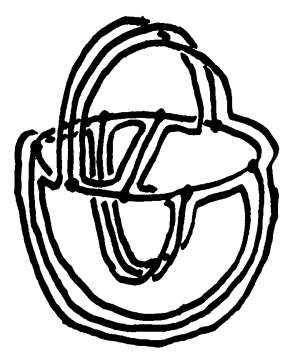

FIGURE 2

I do not know the logical status of the assertion, as I am unable either to prove it or to find a counterexample. Fortunately, for the polynomials which are the subject of this paper, a weaker result will suffice.

DEFORMATION LEMMA. Under the continuing and additional assumptions above there is a homotopy $H: \overline{F_{0}} \times I \rightarrow \bar{F}_{0}$ such that for all $\vec{z}$ in $\overline{F_{0}}$ we have $H(\vec{z}, 0)=\vec{z}$ and $H(\vec{z}, 1) \in G$. Hence if $i: G \rightarrow \bar{F}_{0}$ denotes the inclusion map, then $i_{*}$ induces epimorphisms in homology.

This lemma is true. In fact, I propose to prove it in $\$ \S 4-14$. I hope it is a satisfactory partial response to remark (3.5) on p. 208 of [8].

4. The plan of the proof is to construct $H$ from four homotopies, labeled $H_{1}$ to $H_{4}$, and certain other maps designed to correct the target spaces of the $H_{j}$ 's, viz.,

$$
H=g_{2} \circ g_{1} \circ\left[H_{1} *\left(\rho \circ\left(H_{2} * H_{3}\right)\right)\right] * H_{4} \text {. }
$$

The meaning of the * symbol is as follows. Given spaces $X, Y, Z$, and $W, Y \subseteq W$ $\subseteq Z$, and homotopies $H^{\prime}: X \times I \rightarrow Y$ and $H^{\prime \prime}: Z \times I \rightarrow W$ such that $H^{\prime \prime}(y, 0)=$ $y$ for all $y$ in $Y$, define $H^{\prime} * H^{\prime \prime}: X \times I \rightarrow W$ by

$$
\begin{aligned}
H^{\prime} * H^{\prime \prime}(x, t) & =H^{\prime}(x, 2 t) \text { if } 0 \leqslant t<\frac{1}{2}, \\
& =H^{\prime \prime}\left(H^{\prime}(x, 1), 2 t-1\right) \text { if } \frac{1}{2} \leqslant t \leqslant 1 .
\end{aligned}
$$

In other words, do $H^{\prime}$ first and then $H^{\prime \prime}$.

Recall that $\overline{F_{0}} \subseteq S^{2 m-1} \subseteq \mathbf{C}^{m}$, and that $f=f_{1}+f_{2}+\cdots+f_{r}$, each $f_{j}$ being a monomial.

$H_{1}: \overline{F_{0}} \times I \rightarrow \overline{F_{0}}$ will have the following properties: for all $\vec{z}$ in $\overline{F_{0}}, H_{1}(\vec{z}, 0)=\vec{z}$ and $f\left(H_{1}(\vec{z}, 1)\right) \geqslant c$, where $c$ is a positive real number to be determined in $\S 5$ below.

$H_{2}: \mathbf{C}^{m} \times I \rightarrow C^{m}$ will have the following properties: for all $\vec{z}$ in $\mathbf{C}^{m}, H_{2}(\vec{z}, 0)=$ $\vec{z}$ and for each $j=1,2, \ldots, r$ the real part $\operatorname{Re}\left(f_{j}\left(H_{2}(\vec{z}, t)\right)\right)$ increases as $t$ increases from 0 to 1 until either $\operatorname{Re}\left(f_{j}\left(H_{2}(\vec{z}, 1)\right)\right)>0$ or $\left|f_{j}\left(H_{2}(\vec{z}, 1)\right)\right| \leqslant \delta$, where $\delta$ is a positive number to be determined in $\$ 12$ below; the imaginary parts do not change during $\mathrm{H}_{2}$.

$H_{3}: \mathbf{C}^{m} \times I \rightarrow \mathrm{C}^{m}$ will have the following properties: for all $\vec{z}$ in $\mathbf{C}^{m}, H_{3}(\vec{z}, 0)=$ $\vec{z}$ and for each $j=1,2, \ldots, r$ the absolute value of the imaginary part $\operatorname{Im}\left(f_{j}\left(H_{3}(\vec{z}, t)\right)\right)$ decreases as $t$ increases from 0 to 1 until either $\operatorname{Im}\left(f_{j}\left(H_{3}(\vec{z}, 1)\right)\right)=0$ 
or $\left|f_{j}\left(H_{3}(\vec{z}, 1)\right)\right| \leqslant \delta$; the real parts do not change during $H_{3}$. In addition, for all $\vec{z}$ in $\mathbf{C}^{m}$ such that $\operatorname{Re}(f(\vec{z})) \geqslant c$ the point $H_{2} * H_{3}(\vec{z}, t)$ will lie outside $S^{2 m-1}$.

The map $\rho: \mathbf{C}^{m}-\{0\} \rightarrow S^{2 m-1}$ is simply the normalization: $\rho(\vec{z})=\vec{z} /|\vec{z}|$.

$H_{4}: S^{2 m-1} \times I \rightarrow S^{2 m-1}$ will leave the argument of each coordinate $z_{k}$ (hence of each monomial $f_{j}(\vec{z})$ ) unchanged and will reduce the magnitude of each monomial of magnitude $\delta$ or less to zero, without allowing any such magnitude to get as large as $c / m$ along the way.

Assuming the above premises fulfilled, we will have found a homotopy $\left[H_{1} *\left(\rho \circ\left(H_{2} * H_{3}\right)\right)\right] * H_{4}: \overline{F_{0}} \times I \rightarrow\left\{\vec{z} \in S^{2 m-1}: \operatorname{Re}(f(\vec{z}))>-c\right\}$ such that, for all $\vec{z}$ in $\overline{F_{0}},\left(\left[H_{1} *\left(\rho \circ\left(H_{2} * H_{3}\right)\right)\right] * H_{4}\right)(\vec{z}, 1) \in G$. The final step will be to compose with functions $g_{1}$ and $g_{2}$ so as to change the target space of the homotopy from $\{\operatorname{Re}(f(\vec{z}))>-c\}$ to $\overline{F_{0}}$, i.e., to $\{\operatorname{Re}(f(\vec{z})) \geqslant 0$ and $\operatorname{Im}(f(\vec{z}))=0\}$. The map $g_{1}$ will satisfy $g_{1}:\left\{\vec{z} \in S^{2 m-1}: \operatorname{Re}(f(\vec{z}))>-c\right\} \rightarrow \cup_{-\pi<\theta<\pi} \overline{F_{\theta}}=S^{2 m-1}-F_{\pi}$, and $g_{2}$ will "close the book" (see [3, p. 6] or [7, pp. 705-706]).

5. It is convenient to begin at the end, with $g_{1}$ and $g_{2}$, since the construction of $g_{1}$ dictates a choice of the positive number $c$. Since $\bar{F}_{0}$ is a smooth manifold with boundary, the boundary being $K$, there exists a collaring of $K$, that is, there is an open neighborhood $U$ of $K$ in $\bar{F}_{0}$ and a diffeomorphism $\Phi: K \times[0,1) \rightarrow U$. Hence there is a neighborhood $W$ of $K$ included in $U$, say $W=\Phi\left(K \times\left[0, \frac{1}{2}\right)\right)$, and a smooth homotopy $P: \overline{F_{0}} \times I \rightarrow \bar{F}_{0}$ such that $P(\vec{z}, 0)=\vec{z}$ for all $\vec{z}$ in $\overline{F_{0}}, P(\vec{z}, t)=\vec{z}$ for all $\vec{z}$ in $K$ and all $t$, and $P(\vec{z}, 1) \in K$ for all $\vec{z}$ in $W$. Let $c=\min \{|f(\vec{z})|=f(\vec{z})$ : $\left.\vec{z} \in \overline{F_{0}}-W\right\}$. Then $|f(\vec{z})|<c$ implies $P(\vec{z}, 1) \in K$. (Alternatively, we may take $c$ to be the minimum critical value of $|f|$ on $F_{0}$ and use the Morse theory of $|f|$ to construct $P$; compare [3, pp. 49-50].)

Now for any angle $\theta$, let $h_{\theta}: \overline{F_{0}} \rightarrow \overline{F_{\theta}}$ be the diffeomorphism defined by $h_{\theta}\left(z_{1}, z_{2}, \ldots, z_{m}\right)=\left(z_{1} \cdot \exp \left\{i \theta / w_{1}\right\}, z_{2} \cdot \exp \left\{i \theta / w_{2}\right\}, \ldots, z_{m} \exp \left\{i \theta / w_{m}\right\}\right) . W e$ will make our two retractions $g_{1}$ and $g_{2}$ from $P$ by using the family $\left\{h_{\theta}\right\}$.

First, let $X=\cup\left\{\bar{F}_{\theta}: \pi / 2 \leqslant \theta \leqslant 3 \pi / 2\right\}$ and define $p_{1}: X \rightarrow X$ by $p_{1}(\vec{z})=$ $h_{\theta}\left(P\left(h_{-\theta}(\vec{z}),|\cos \theta|\right)\right)$ if $\vec{z} \in \overline{F_{\theta}}$. Then define $g_{1}:\left\{\vec{z} \in S^{2 m-1}: \operatorname{Re}(f(\vec{z}))>-c\right\} \rightarrow$ $\bigcup\left\{\bar{F}_{\theta}:-\pi<\theta<\pi\right\}$ by setting $g_{1}(\vec{z})=p_{1}(\vec{z})$ if $\vec{z} \in \cup\left\{\bar{F}_{\theta}: \pi / 2<\theta<3 \pi / 2\right\}$ and $g_{1}(\vec{z})=\vec{z}$ otherwise.

Second, define $p_{2}(\vec{z})=P(\vec{z}, 1)$ for $\vec{z}$ in $\overline{F_{0}}$ and define $g_{2}: \cup\left\{\overline{F_{\theta}}:-\pi<\theta<\pi\right\}$ $\rightarrow \overline{F_{0}}$ as follows:

$$
\begin{array}{ll}
g_{2}(\vec{z})=h_{(1-u) \theta} \circ p_{2} \circ h_{-\theta}(\vec{z}) & \text { if } \vec{z} \in \overline{F_{\theta}} \text { and }|f(\vec{z})|=c \cdot u, 0<u \leqslant 1 ; \\
g_{2}(\vec{z})=p_{2} \circ h_{-\theta}(\vec{z}) & \text { if } \vec{z} \in \overline{F_{\theta}} \text { and }|f(\vec{z})|>c .
\end{array}
$$

I leave it to the reader to verify that the formulas for $g_{1}$ and $g_{2}$ are unambiguous and yield continuous functions with the desired properties.

6. Construction of $H_{1}$. This is closely related to the collaring step in $\S 5$. Making use of the same notatioin we could for instance define

$$
\begin{array}{ll}
H_{1}(\vec{z}, t)=\Phi\left(\vec{z}^{\prime}, \max \{(1-t) u+t / 2, u\}\right) & \begin{array}{l}
\text { if } \vec{z}=\Phi\left(\vec{z}^{\prime}, u\right) \text { for some } \vec{z} \text { in } K \\
\text { and some } u \text { in }[0,1)
\end{array} \\
H_{1}(\vec{z}, t)=\vec{z} & \text { otherwise. }
\end{array}
$$


7. Construction of $\mathrm{H}_{2}$ (heuristics). Assume we are given a positive number $\delta$ such that $m \delta \leqslant c$. (See $\S 12$ below for the construction of $\delta$.) The homotopy $\mathrm{H}_{2}: \mathrm{C}^{m} \times I$ $\rightarrow \mathbf{C}^{m}$ is a flow which integrates a certain system of differential equations. Recall that our polynomial $f$ is a sum of $r$ monomials $f_{1}, f_{2}, \ldots, f_{r}$ whose exponent vectors $\left(e_{j, 1}, e_{j, 2}, \ldots, e_{j, m}\right)$ for $j=1,2, \ldots, r$ form an independent set. By adding to $f$ a total of $m-r$ more monomials with coefficient $c_{j}=0$ we may without altering any of the sets $F_{\theta}, K, G$, etc., arrange that $r=m$. Let $E$ be the invertible matrix whose entries are the $e_{j, k}$ 's, and let $\left(E^{-1}\right)_{j, k}$ denote a typical entry of the inverse matrix. Fix $\vec{z}$ in $\mathbf{C}^{m}$ and write $\vec{p}(t)$ for $\mathrm{H}_{2}(\vec{z}, t)$. If we could arrange that the time derivative of $f_{j}(\vec{p}(t))$ should satisfy $d f_{j}(\vec{p}(t)) / d t=\left|f_{j}(\vec{p}(t))\right|^{2} \alpha_{j}$ for some nonnegative real number $\alpha_{j}$ (depending on $\vec{z}$ ), then in unit time the real part of $f_{j}$ would increase a distance determined by $\alpha_{j}$ and $\vec{z}$, while the imaginary part would remain stationary. This leads as we shall see to the system of differential equations

$$
\frac{d}{d t} p_{j}(t)=p_{j}(t) \sum_{k=1}^{m}\left(E^{-1}\right)_{j, k} \alpha_{k}(\vec{z}) \overline{f_{k}(\vec{p}(t))},
$$

where $p_{j}$ denotes the $j$ th coordinate of $\vec{p}$. Unfortunately, for a satisfactory choice of parameters $\left\{\alpha_{k}\right\}$, this system does not have compact support, and so the method which follows is the grubby one of producing explicit solutions.

8. Let us begin by establishing goals for the real parts of the various monomials to reach in unit time, and then computing the associated velocity multipliers $\alpha_{k}(\vec{z})$.

Choose a positive real number $M$ such that $\left|f_{k}(\vec{z})\right| \leqslant M$ for all $\vec{z}$ in $S^{2 m-1}$ and $k=1,2, \ldots, m$, and so that $M \geqslant 2 \delta$. Define $g: \mathbf{C} \rightarrow \mathbf{R}$ (" $g$ " for "goal") as follows. For $z$ in $\mathbf{C}$, write $z=x+i y, x, y \in \mathbf{R}$.

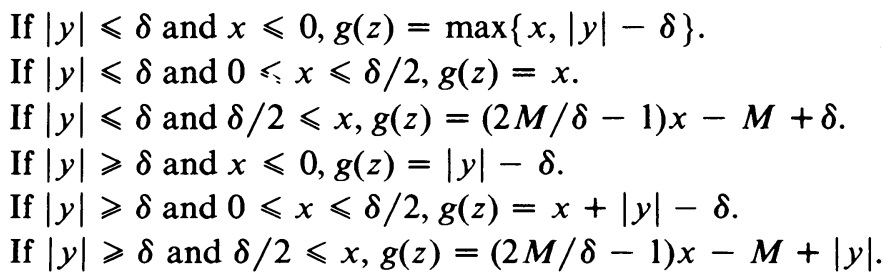

Here are some elementary properties of $g$ :

(i) $g$ is continuous;

(ii) for all $z, g(z) \geqslant x$;

(iii) for all $z, g(z)>0$ or $|(g(z), y)| \leqslant \delta$;

(iv) $x \geqslant \delta$ implies $g(z) \geqslant M$;

(v) if $y=0$ and $x \neq 0$, then $x$ and $g(z)$ have the same sign.

Define $\alpha: \mathbf{C} \rightarrow \mathbf{R}^{+}$as follows:

$\alpha(0)=0$.

If $z=x+i y \neq 0, \alpha(z)=\int_{x}^{g(z)} d u /\left(u^{2}+y^{2}\right)$.

This is legal by property (v) above. Continuity of $\alpha$ at 0 follows from the fact that $g(z)=x$ whenever $|x|+|y| \leqslant \delta / 2$. Continuity away from 0 follows from an elementary estimate.

Define $\alpha_{k}: \mathbf{C}^{m} \rightarrow \mathbf{R}^{+}$by $\alpha_{k}(\vec{z})=\alpha\left(f_{k}(\vec{z})\right)$ for $k=1,2, \ldots, m$. 
9. The $k$ th component of $H_{2}$ will have the form $r_{k} \exp \left\{i \theta_{k}\right\}$. Also entering into the formulas will be the arguments $\left\{\psi_{k}\right\}$ of the various monomials; that is, $f_{k}=\left|f_{k}\right| \exp \left\{i \psi_{k}\right\}$.

Let us begin with $\theta_{k}:\left\{\vec{z} \in \mathbf{C}^{m}: z_{k} \neq 0\right\} \times \mathbf{R} \rightarrow \mathbf{R} / 2 \pi Z$ for $k=1,2, \ldots, m$. Write $z_{k}=\left|z_{k}\right| \exp \left\{i a_{k}\right\}$ and set

$$
\theta_{k}(\vec{z}, t)=a_{k}-t \sum_{j=1}^{m}\left(E^{-1}\right)_{k, j} \cdot \alpha_{j}(\vec{z}) \cdot \operatorname{Im}\left(f_{j}(\vec{z})\right) .
$$

If the coefficient $c_{k}$ is not zero, write $c_{k}=\left|c_{k}\right| \exp \left\{i b_{k}\right\}$ and define an argument function $\psi_{k}: \mathbf{C}^{m} \times \mathbf{R} \rightarrow \mathbf{R} / 2 \pi Z$ by

$$
\psi_{k}(\vec{z}, t)=b_{k}+\sum_{\left\{l: z_{l} \neq 0\right\}} e_{k, l} \cdot \theta_{l}(\vec{z}, t) .
$$

Suppose $f_{k}(\vec{z}) \neq 0$. Then there is no $l$ such that $e_{k, l}>0$ and $z_{l}=0$. Also $c_{k} \neq 0$, so $\psi_{k}$ is defined. Furthermore, $f_{k}(\vec{z})=\left|f_{k}(\vec{z})\right| \exp \left\{i \psi_{k}(\vec{z}, 0)\right\}$ and $\psi_{k}(\vec{z}, t)=\psi_{k}(\vec{z}, 0)-$ $\alpha_{k}(\vec{z}) \cdot \operatorname{Im}\left(f_{k}(\vec{z})\right) \cdot t$. Observe that if $f_{k}(\vec{z})$ is a positive real number, then $f_{k}(\vec{z}) \cdot \alpha_{k}(\vec{z})$ $<1$; in fact, $\alpha_{k}(\vec{z})=\left(1 / f_{k}(\vec{z})\right)-\left(1 / g\left(f_{k}(\vec{z})\right)\right)$. Also, if $f_{k}(\vec{z})$ is not real, then the numbers $\sin \psi_{k}(\vec{z}, t)$ and $\sin \psi_{k}(\vec{z}, 0)$ have the same sign for all $t$ in $[0,1]$. To see this, let $f_{k}(\vec{z})=x+i y, x, y \in \mathbf{R}$. Assume $y \neq 0$. Then

$$
\alpha_{k}(\vec{z})=y^{-1}\{\arctan (g(x+i y) / y)-\arctan (x / y)\}
$$

so $\alpha_{k}(\vec{z}) \cdot \operatorname{Im}\left(f_{k}(\vec{z})\right)=(\operatorname{sgn} y) \cdot$ the smallest positive angle from $(x, y)$ to $(g(x+i y), y)]$. Since $\psi_{k}(\vec{z}, 0)$ is an argument for $f_{k}(\vec{z})$, the assertion follows.

Define $r_{k}: \mathbf{C}^{m} \times[0,1] \rightarrow \mathbf{R}^{+}$as follows.

If $z_{k}=0$, set $r_{k}(\vec{z}, t)=0$ for all $t$. If $z_{k} \neq 0$ set

$$
\begin{aligned}
r_{k}(\vec{z}, t)=\left|z_{k}\right| & \prod_{\left\{l: f_{l}(\vec{z}) \in R\right\}}\left[1-\alpha_{l}(\vec{z}) \cdot f_{l}(\vec{z}) \cdot t\right]^{-\left(E^{-1}\right) k, l} \\
& \prod_{\left\{l: f_{l}(\vec{z}) \notin R\right\}}\left[\sin \psi_{l}(z, t) / \sin \psi_{l}(z, 0)\right]^{-\left(E^{-1}\right) k, l} .
\end{aligned}
$$

Define $H_{2}: \mathbf{C}^{m} \times[0,1] \rightarrow \mathbf{C}^{m}, H_{2}=\left(H_{2}^{(1)}, H_{2}^{(2)}, \ldots, H_{2}^{(m)}\right)$, by setting

$$
\begin{aligned}
& H_{2}^{(k)}(\vec{z}, t)=0 \quad \text { for all } t \text { if } z_{k}=0, \\
& H_{2}^{(k)}(\vec{z}, t)=r_{k}(\vec{z}, t) \exp \left\{i \theta_{k}(\vec{z}, t)\right\} \quad \text { if } z_{k} \neq 0 .
\end{aligned}
$$

Here are some properties of $H_{2}$.

(i) $H_{2}(\vec{z}, 0)=\vec{z}$.

(ii) If $f_{k}(\vec{z}) \in \mathbf{R}$, then $f_{k}\left(H_{2}(\vec{z}, t)\right)=f_{k}(\vec{z}) /\left[1-\alpha_{k}(\vec{z}) \cdot f_{k}(\vec{z}) \cdot t\right]$.

(iii) If $f_{k}(\vec{z}) \notin \mathbf{R}$, then

$$
\begin{aligned}
f_{k}\left(H_{2}(\vec{z}, t)\right) & =f_{k}(\vec{z}) \cdot\left[\sin \psi_{k}(\vec{z}, 0) / \sin \psi_{k}(\vec{z}, t)\right] \cdot \exp \left\{-i \alpha_{k}(\vec{z}) \cdot \operatorname{Im}\left(f_{k}(\vec{z})\right) \cdot t\right\} \\
& =\left|f_{k}(\vec{z})\right| \exp \left\{i \psi_{k}(\vec{z}, t)\right\} \cdot \sin \psi_{k}(\vec{z}, 0) / \sin \psi_{k}(\vec{z}, t) \\
& =\operatorname{Im}\left(f_{k}(\vec{z})\right)\left[\cot \psi_{k}(\vec{z}, t)+i\right] .
\end{aligned}
$$

Given $\vec{z}$, denote $H_{2}(\vec{z}, t)$ by $\vec{p}(t)$, as in $\S 7$, and write $\vec{p}(t)=\left(p_{1}(t), \ldots, p_{m}(t)\right)$. Then

$$
\frac{d}{d t} p_{j}(t)=p_{j}(t) \sum_{k=1}^{m}\left(E^{-1}\right)_{j, k} \cdot \alpha_{k}(\vec{z}) \cdot \overline{f_{k}(\vec{p}(t))} .
$$


To see this, just differentiate, observing that by properties (ii) and (iii) above, $\operatorname{Im}\left(f_{k}(\vec{p}(t))\right)=\operatorname{Im}\left(f_{k}(\vec{z})\right)$ for all $t$. Since the right-hand sides of these differential equations are smooth functions of $p_{1}, \ldots, p_{m}$, it follows from the general theory of ordinary differential equations that $\mathrm{H}_{2}$ is continuous on $\mathrm{C}^{m} \times[0,1]$ (see, for example, [2, Chapter IX, Theorem 4, pp. 156-157]).

Following Milnor [3, p. 33], for an analytic function $h\left(z_{1}, \ldots, z_{m}\right)$ define $\overrightarrow{\operatorname{grad} h}=\left(\overline{\partial h / \partial z_{1}}, \ldots, \overline{\partial h / \partial z_{m}}\right)$, so that the chain rule reads $d h(\vec{p}(t)) / d t=$ $\langle d \vec{p} / d t, \overrightarrow{\operatorname{grad}} h\rangle$. An easy calculation now shows that $\left\langle d \vec{p} / d t, \overrightarrow{\operatorname{grad}} f_{k}(\vec{p}(t))\right\rangle=$ $\left|f_{k}(\vec{p}(t))\right|^{2} \cdot \alpha_{k}(\vec{z})$. Since this is a real number, it follows that it is the rate of change of the real part of $f_{k}(\vec{p}(t))$ and (a fact already noted above) that $\operatorname{Im}\left(f_{k}(\vec{p}(t))\right)$ does not change with $t$. The functions $\alpha_{k}$ were concocted precisely so that in unit time the real part of $f_{k}$ would reach the goal $g\left(f_{k}(\vec{z})\right)$, defined in $\S 8$.

Hence as $t$ goes from 0 to $1, \operatorname{Re}\left(f_{k}\left(H_{2}(\vec{z}, t)\right)\right)$ increases until either it is positive or $\left|f_{k}\left(H_{2}(\vec{z}, 1)\right)\right| \leqslant \delta$.

10. Construction of $H_{3}$. This is very similar to the construction of $H_{2}$. In brief, here are the formulas.

Define $g^{\prime}: \mathbf{C} \rightarrow \mathbf{R}$ as follows:

$$
\begin{aligned}
& g^{\prime}(x+i y)=\min \{0, \max \{y,|x|-\delta\}\} \quad \text { if } y \leqslant 0 \\
& g^{\prime}(x+i y)=\max \{0, \min \{y, \delta-|x|\}\} \quad \text { if } y>0 .
\end{aligned}
$$

Define $\alpha^{\prime}: \mathbf{C} \rightarrow \mathbf{R}$ by setting $\alpha^{\prime}(0)=0$ and $\alpha^{\prime}(x+i y)=\int_{y}^{g^{\prime}(x+i y)} d u /\left(x^{2}+u^{2}\right)$ if $x+i y \neq 0$. Then set $\alpha_{k}^{\prime}(\vec{z})=\alpha^{\prime}\left(f_{k}(\vec{z})\right)$. Note that in contrast to the case of $H_{2}$ the goals $g^{\prime}$ may be less than the initial imaginary parts $y$, and hence the multipliers $\alpha_{k}^{\prime}$ may be negative.

Write $z_{k}=\left|z_{k}\right| \exp \left\{i a_{k}\right\}$ and define $\theta_{k}^{\prime}:\left\{\vec{z} \in \mathbf{C}^{m}: z_{k} \neq 0\right\} \times \mathbf{R} \rightarrow \mathbf{R} / 2 \pi Z$ by setting

$$
\theta_{k}^{\prime}(z, t)=a_{k}+t \cdot \sum_{j=1}^{m}\left(E^{-1}\right)_{k, j} \cdot \alpha_{j}^{\prime}(\vec{z}) \cdot \operatorname{Re}\left(f_{j}(\vec{z})\right) .
$$

If $c_{k} \neq 0$, write $c_{k}=\left|c_{k}\right| \exp \left\{i b_{k}\right\}$ and define $\psi_{k}^{\prime}: \mathbf{C}^{m} \times \mathbf{R} \rightarrow \mathbf{R} / 2 \pi Z$ by the formula

$$
\psi_{k}^{\prime}(\vec{z}, t)=b_{k}+\sum_{\left\{l: z_{l} \neq 0\right\}} e_{k, l} \cdot \theta_{l}^{\prime}(\vec{z}, t) .
$$

Define $r_{k}^{\prime}: \mathbf{C}^{m} \times[0,1] \rightarrow \mathbf{R}^{+}$as follows: if $z_{k}=0$, set $r_{k}^{\prime}(\vec{z}, t)=0$ for all $t$; if $z_{k} \neq 0$, set

$$
\begin{aligned}
r_{k}^{\prime}(\vec{z}, t)= & \left|z_{k}\right| \cdot \prod_{\left\{l: f_{l}(\vec{z}) \in i R\right\}}\left[1+i \alpha_{l}^{\prime}(\vec{z}) \cdot f_{l}(\vec{z}) \cdot t\right]^{-\left(E^{-1}\right) k, l} \\
& \cdot \prod_{\left\{l: f_{l}(\vec{z}) \notin i R\right\}}\left[\cos \psi_{l}^{\prime}(\vec{z}, t) / \cos \psi_{l}^{\prime}(\vec{z}, 0)\right]^{-\left(E^{-1}\right) k, l} .
\end{aligned}
$$

Define $H_{3}^{(k)}: \mathbf{C}^{m} \times[0,1] \rightarrow \mathbf{C}$ by setting

$$
H_{3}^{(k)}(\vec{z}, t)=0 \text { for all } t \text { if } z_{k}=0,
$$


and

$$
H_{3}^{(k)}(\vec{z}, t)=r_{k}^{\prime}(\vec{z}, t) \cdot \exp \left\{i \theta_{k}^{\prime}(\vec{z}, t)\right\} \quad \text { if } z_{k} \neq 0 .
$$

Then set $H_{3}=\left(H_{3}^{(1)}, H_{3}^{(2)}, \ldots, H_{3}^{(m)}\right)$.

This time we have the differential equations

$$
\frac{\partial}{\partial t} H_{3}^{(k)}(\vec{z}, t)=H_{3}^{(k)}(\vec{z}, t) \sum_{l=1}^{m} i\left(E^{-1}\right)_{k, l} \cdot \alpha_{l}^{\prime}(\vec{z}) \overline{f_{l}\left(H_{3}(\vec{z}, t)\right)},
$$

and hence

$$
\left\langle\frac{\partial}{\partial t} H_{3}(\vec{z}, t), \overrightarrow{\operatorname{grad}} f_{k}\left(H_{3}(\vec{z}, t)\right)\right\rangle=i \alpha_{k}^{\prime}(\vec{z}) \cdot\left|f_{k}\left(H_{3}(\vec{z}, t)\right)\right|^{2} .
$$

Thus while the real part of $f_{k}\left(H_{3}(\vec{z}, t)\right)$ remains constant in $t$, the imaginary part has time derivative $\alpha_{k}^{\prime}(\vec{z}) \cdot\left|f_{k}\left(H_{3}(\vec{z}, t)\right)\right|^{2}$. Again, $\alpha_{k}^{\prime}$ is concocted so that the imaginary part of $f_{k}$ reaches the goal $g^{\prime}\left(f_{k}(\vec{z})\right)$; hence for all $\vec{z}$ either $\operatorname{Im}\left(f_{k}\left(H_{3}(\vec{z}, 1)\right)\right)=0$ or $\left|f_{k}\left(H_{3}(\vec{z}, 1)\right)\right| \leqslant \delta$.

11. At this stage we have a homotopy $H_{2} * H_{3}: \mathbf{C}^{m} \times I \rightarrow \mathbf{C}^{m}$ which among other things increases the real parts of all the monomials of $f$, and hence increases the real part of $f$. Note next that if $f(\vec{z})$ is real and $f(\vec{z})>c$, then $\left(H_{2} * H_{3}\right)(\vec{z}, 1)$ lies outside the sphere $S^{2 m-1}$. To see this, observe that since $m \delta<c$ (see $\S 12$ ), at least one of the monomials $f_{k}(\vec{z})$ must have real part at least $\delta$. From property (iv) of $\S 8$, we have then that $\operatorname{Re}\left(f_{k}\left(H_{2}(\vec{z}, 1)\right)\right) \geqslant M$. Because $H_{3}$ does not alter the real parts of the monomials, it is therefore also the case that $\operatorname{Re}\left(f_{k}\left(H_{2} * H_{3}(\vec{z}, 1)\right)\right) \geqslant M$, and hence that $\left|f_{k}\left(H_{2} * H_{3}(\vec{z}, 1)\right)\right| \geqslant M$. By choice of $M$, however, $\left|f_{k}\right|$ does not exceed $M$ on $S^{2 m-1}$, and so the assertion follows. Therefore, when we follow $\mathrm{H}_{2} * \mathrm{H}_{3}$ with the normalization map $\rho: \mathbf{C}^{m}-\{0\} \rightarrow S^{2 m-1}$ defined by $\rho(\vec{z})=\vec{z} /|\vec{z}|$, and restrict the composite $\rho \circ\left(H_{2} * H_{3}\right)$ to pairs $(\vec{z}, t)$ for which $f(\vec{z})$ is real and $f(\vec{z})>c$, then at the point $\rho \circ\left(H_{2} * H_{3}\right)(\vec{z}, 1)$ every monomial $f_{k}$ either has magnitude less than $\delta$ or has positive real part and zero imaginary part.

12. Definition of $\delta$. Denote by $S^{+}$the portion $\left\{\left(r_{1}, r_{2}, \ldots, r_{m}\right) \in S^{m-1} \subseteq \mathbf{R}^{m}: 0\right.$ $\leqslant r_{k}$ for all $\left.k\right\}$ of the (real) $(m-1)$-sphere. It will be convenient to deal also with the simplex $\Delta=\left\{\left(r_{1}, r_{2}, \ldots, r_{m}\right) \in \mathbf{R}^{m}: 0 \leqslant r_{k}\right.$ for all $k$ and $\left.r_{1}+\cdots+r_{m}=1\right\}$. Let $\rho_{0}: \Delta \rightarrow S^{+}$be the homeomorphism defined by projecting from the origin. For $\vec{r}$ in $S^{+}$we will be considering real monomial values $\left|f_{k}(\vec{r})\right|=\left|c_{k}\right|$. $r_{1}^{e_{k, 1}} r_{2}^{e_{k, 2}} \cdots r_{m}^{e_{k, m}}$.

For $k=1,2, \ldots, m$ define $G_{k}=\left\{\vec{r} \in \Delta:\left|f_{k}\left(\rho_{0}(\vec{r})\right)\right|>c / m\right\}$. (The number $c$ was determined in $\S 5$ above.) Then $G_{k}$ is a compact subset of $\Delta$ which for each index $j$ such that $e_{k, j}>0$ does not meet the $j$ th face of $\Delta,\left\{\vec{r} \in \Delta: r_{j}=0\right\}$. Hence there exists a positive number $\eta$ such that, for each $k, G_{k}$ is included in the set $\left\{\vec{r} \in \Delta: r_{j} \geqslant \eta\right.$ if $\left.e_{k_{j}}>0\right\}$. Call this set $\Delta(k)$; it is a simplex included in and smaller than $\Delta$ with faces parallel to those of $\Delta$. We may assume that $\eta<1 / m$, so that for each $k, \Delta(k)$ contains the barycenter of $\Delta$ in its interior.

If $c_{k} \neq 0$, let $\delta_{k}=\min \left\{\left|f_{k}\left(\rho_{0}(\vec{r})\right)\right|: \vec{r} \in \Delta(k)\right\}$. Then $\delta_{k}>0$, since $f_{k}\left(\rho_{0}(\vec{r})\right) \neq 0$ for $\vec{r}$ in $\Delta(k)$. Note also that $\delta_{k} \leqslant c / m$. If $c_{k}=0$ we do not define $\delta_{k}$. Let $\delta$ be the minimum of the numbers $\delta_{k}$ for those $k$ such that $c_{k} \neq 0$. Note that $m \delta \leqslant c$. 
Before constructing $H_{4}$, let us record for later use the crucial properties of $\delta$ and $\eta$. Let $\vec{r}$ denote $\left(r_{1}, r_{2}, \ldots, r_{m}\right) \in \Delta$.

(i) For $k=1,2, \ldots, m$, if $\left|f_{k}\left(\rho_{0}(\vec{r})\right)\right| \leqslant \delta$, then for some index $j$ we have $e_{k, j}>0$ and $r_{j}<\eta$.

(ii) For $k=1,2, \ldots, m$, if for some index $j$ we have $e_{k, j}>0$ and $r_{j}<\eta$, then $\left|f_{k}\left(\rho_{0}(\vec{r})\right)\right|<c / m$.

13. Construction of $H_{4}$. For $\left(z_{1}, z_{2}, \ldots, z_{m}\right)$ in $S^{2 m-1}$ write $r_{j}=\left|z_{j}\right|$ for each $j$, so that $\left(r_{1}, r_{2}, \ldots, r_{m}\right) \in S^{+}$. The plan is to construct a homotopy $Q=$ $\left(Q_{1}, Q_{2}, \ldots, Q_{m}\right): \Delta \times I \rightarrow \Delta$ with the properties:

(i) $Q(\vec{r}, 0)=\vec{r}$;

(ii) if $r_{j}=0$, then $Q_{j}(\vec{r}, t)=0$ for all $t$;

(iii) if $r_{j}<\eta$, then $Q_{j}(\vec{r}, t)$ decreases as $t$ increases, and so $Q_{j}(\vec{r}, t)<\eta$ for all $t$;

(iv) if $r_{j}<\eta$, then $Q_{j}(\vec{r}, 1)=0$.

Let us connote properties (ii), (iii), and (iv) briefly by saying that $Q$ "wipes out small coordinates".

Once we have $Q$ we can define $H_{4}: S^{2 m-1} \times I \rightarrow S^{2 m-1}$ as follows. Write $z_{j}=r_{j} \exp \left\{i \theta_{j}\right\}$, using an arbitrary value of $\theta_{j}$ if $r_{j}=0$. Then the $j$ th component of $H_{4}(\vec{z}, t)$ is $\rho_{0} \circ Q_{j}\left(\rho_{0}^{-1}(\vec{r}), t\right) \cdot \exp \left\{i \theta_{j}\right\}$. Property (ii) of $Q$ guarantees the continuity of $H_{4}$. Properties (iii) and (iv), together with the two properties in $\S 12$ of the numbers $\delta$ and $\eta$, imply that $H_{4}$ wipes out each monomial which begins with magnitude not exceeding $\delta$, while never allowing the magnitude to reach $\mathrm{c} / \mathrm{m}$ during the homotopy.

14. The construction of $Q$ is by induction on the skeletons of $\Delta$. Let $\Delta^{(k)}$ denote the $k$-skeleton. Given a nonempty subset $A$ of the set $\{1,2, \ldots, m\}$ of indices, define the face $\Delta_{A}$ by $\Delta_{A}=\left\{\vec{r} \in \Delta: r_{j}=0\right.$ if $\left.j \notin A\right\}$. Let $Q_{A}: \Delta_{A} \times I \rightarrow \Delta_{A}$ be a homotopy beginning at the identity map such that whenever $\vec{r}$ in $\Delta_{A}$ has the property that $r_{j}<\eta$ for some $j$ in $A$, then $Q_{A}(\vec{r}, t)$ lies on the ray from the barycenter of $\Delta_{A}$ through $\vec{r}$, increasingly far beyond $\vec{r}$ as $t$ increases and on the boundary $\partial \Delta_{A}$ when $t=1$. In other words, $Q_{A}$ is a radial expansion which pushes to the boundary all points that are sufficiently close to the boundary initially. Let us record some elementary properties of $Q_{A}$.

(i) $Q_{A}$ leaves $\partial \Delta_{A}$ fixed.

(ii) If $\vec{r} \in \Delta_{A}$ and $r_{j}<\eta$, then the $j$ th coordinate of $Q_{A}(\vec{r}, t)$ decreases as $t$ increases. Hence it is less than $\eta$ for all $t$.

(iii) If $\vec{r} \in$ Int $\Delta_{A}$ and for some $j$ we have $r_{j}<\eta$, then $Q_{A}(\vec{r}, 1)$ is in a lower skeleton of $\Delta$ than $\vec{r}$ (although if $A$ has more than two members it is not necessarily true that the $j$ th coordinate of $Q_{A}(\vec{r}, 1)$ is zero).

On the one-skeleton $\Delta^{(1)}$, define $Q^{(1)}$ by setting $Q^{(1)}(\vec{r}, t)=Q_{A}(\vec{r}, t)$ if $|A|=2$ and $\vec{r} \in \Delta_{A}$. Property (i) above assures that this is unambiguous. It is easy to verify that $Q^{(1)}$ wipes out small coordinates in the sense of $\S 13$.

Assume that we have defined a homotopy $Q^{(k)}: \Delta^{(k)} \times I \rightarrow \Delta^{(k)}$ which begins at the identity and wipes out small coordinates. Given any subset $A$ of $\{1,2, \ldots, m\}$ with $k+2$ elements, we can extend the restriction $Q^{(k)} \mid \partial \Delta_{A}$ to a homotopy 
$\Delta_{A} \times I \rightarrow \Delta_{A}$ of the identity map by the homotopy extension property of simplicial complexes, and thus we can extend $Q^{(k)}$ to a homotopy $Q^{\prime}: \Delta^{(k+1)} \times I \rightarrow \Delta^{(k+1)}$ of the identity map with the property that $Q^{\prime}$ maps $\Delta_{A} \times I$ into $\Delta_{A}$ whenever $|A|=k+2$. Define $Q^{\prime \prime}: \Delta^{(k+1)} \times I \rightarrow \Delta^{(k+1)}$ by setting $Q^{\prime \prime}(\vec{r}, t)=Q_{A}(\vec{r}, t)$ if $|A|=k+2$ and $\vec{r} \in \Delta_{A}$. By property (i) again, $Q^{\prime \prime}$ is unambiguously defined by this formula and does not move $\Delta^{(k)}$. Now define $Q^{(k+1)}: \Delta^{(k+1)} \times I \rightarrow \Delta^{(k+1)}$ by $Q^{(k+1)}=Q^{\prime \prime} * Q^{\prime}$ (see $\S 4$ for this notation). I leave it to the reader to verify that $Q^{(k+1)}$ wipes out small coordinates.

This completes the inductive step. Setting $Q=Q^{(m)}$ finishes the construction of the homotopy $H$ and thus proves the Deformation Lemma enunciated in $\$ 3$.

15. For the rest of this paper, assume that

$$
f\left(z_{1}, z_{2}, \ldots, z_{m}\right)=z_{1}^{a_{1} z_{2}}+z_{2}^{a_{2} z_{3}}+\cdots+z_{m-1}^{a_{m-1}} z_{m}+z_{m}^{a_{m}} z_{1}
$$

and that $a_{1} a_{2} \cdots a_{m}>1$. The goal in what follows is to learn enough about the integral homology of the subset $G$ of $S^{2 m-1}$ where each monomial of $f$ takes on only nonnegative real values so that we can apply the lemma of $\$ 3$. The method is to filter the space $G$ by the number of nonzero coordinates and then to study the associated spectral sequence in total degree $m-1$. It will turn out that the only contributions to $H_{m-1} G$ are in filtration $m$ and, if $m$ is even, $m / 2$.

As a first step, let us decompose $G$ as a sort of cell complex, built up by attaching not ordinary cells or simplices but products of simplices and tori. Let us adopt the convention that references to integral subscripts on such variables as $z_{k}, r_{k}, \theta_{k}$, etc., are to be interpreted modulo $m$. For example, $z_{m+1}=z_{1}$.

For any positive integer $j$ let $\Delta^{j}$ denote the not-quite-standard $j$-simplex $\{\vec{r} \in$ $S^{j}: r_{k} \geqslant 0$ for all $\left.k\right\}$. Also, for any proper subset $A$ of $\{1,2, \ldots, m\}$ define $\Delta_{A}=\left\{\vec{r} \in \Delta^{m-1}: r_{k}=0\right.$ if $\left.k \notin A\right\}$. (These definitions are different from the ones adopted in $\S 12$ in that the new $\Delta_{A}$ is the image under the radial projection homeomorphism $\rho_{0}$ of the old $\Delta_{A}$.) Then $\Delta_{A}$ is a simplex of dimension $|A|-1$. Let $\zeta_{A}: \Delta^{|A|-1} \rightarrow \Delta_{A}$ be the homeomorphism obtained by inserting zeros among the coordinates of $\Delta^{|A|-1}$ at the locations indexed by the complement of $A$.

For any positive integer $j$ let $T^{j}$ denote the standard torus $\left(S^{1}\right)^{\times j}$. Given any proper subset $A$ of $\{1,2, \ldots, m\}$, let us agree to say that $A$ has a block beginning at $k+1$ if $k \notin A$ but $k+1 \in A$. For example, if $m \notin A$ but $1 \in A$, then $A$ has a block beginning at 1. Define $T_{A}=\left\{\vec{w} \in T^{m}\right.$ : for all $k$, if $A$ does not have a block beginning at $k+1$ then $\left.w_{k+1}=w_{k}^{-a_{k}}\right\}$. Let $b(A)$ denote the number of blocks in $A$, i.e., the number of members $k+1$ of $\{1,2, \ldots, m\}, 0 \leqslant k \leqslant m-1$, such that $A$ has a block beginning at $k+1$. Then $T_{A}$ is homeomorphic to $T^{b(A)}$. To avoid ambiguities inherent in the cyclic use of subscripts, let us fix a homeomorphism $\eta_{A}$ : $T^{b(A)} \rightarrow T_{A}$ defined by sending $w$ in $T^{b(A)}$ to the $m$-tuple in $T_{A}$ whose $\left(k_{j}+1\right)$ st coordinate is $w_{j}$, where $0 \leqslant k_{1}<k_{2}<\cdots<k_{b(A)} \leqslant m-1$ and $A$ has a block beginning at $k_{j}+1$ for each $j=1,2, \ldots, b(A)$.

Define $\phi_{A}: \Delta_{A} \times T_{A} \rightarrow S^{2 m-1}$ by

$$
\phi_{A}(\vec{r}, \vec{w})=\left(r_{1} w_{1}, r_{2} w_{2}, \ldots, r_{m} w_{m}\right) .
$$


The purpose of defining $T_{A}$ as above is to ensure that each monomial of $f$ will have only nonnegative real values on the image of $\phi_{A}$. Hence actually $\phi_{A}: \Delta_{A} \times T_{A} \rightarrow G$. Define $\psi_{A}: \Delta^{|A|-1} \times T^{b(A)} \rightarrow G$ by $\psi_{A}=\phi_{A} \circ\left(\zeta_{A} \times \eta_{A}\right)$. Here are three elementary properties of the maps $\psi_{A}$.

(i) $\psi_{A}$ is a homeomorphism on Int $\Delta^{|A|-1} \times T^{b(A)}$.

(ii) $\psi_{A}$ (Int $\left.\Delta^{|A|-1} \times T^{b(A)}\right) \cap \psi_{B}$ (Int $\left.\Delta^{|B|-1} \times T^{b(B)}\right)$ is empty if $A \neq B$.

(iii) $\left\{\vec{z} \in G: z_{k}=0\right.$ for at least one $\left.k\right\}=\bigcup\left\{\right.$ Image $\psi_{A}: A$ is a proper subset of $\{1,2, \ldots, m\}\}$.

16. Suppose $k \notin A$ and $k+l+1 \notin A$, but $k+1 \in A, k+2 \in A, \ldots, k+l$ $\in A$, and suppose $l \geqslant 2$. Let us connote this information by saying that $A$ has a long block beginning at $k+1$ and ending at $k+l$. Assume that $k=k_{j}$ in the notation of $\S 15$, and let $\pi: T^{b(A)} \rightarrow T^{b(A)}$ be the map which sends $\left(w_{1}, w_{2}, \ldots, w_{b(A)}\right)$ to $\left(w_{1}, \ldots, w_{j-1}, w_{j}^{-a_{k+1}}, w_{j+1}, \ldots, w_{b(A)}\right)$. Assume also that the $(k+1)$ st coordinate of $\zeta_{A}\left(r_{1}, r_{2}, \ldots, r_{|A|}\right)$ is $r_{u}$, that is, $\zeta_{A}$ inserts $k+1-u$ zeros ahead of $r_{u}$. Define $i_{1}: \Delta^{|A|-2} \rightarrow \Delta^{|A|-1}$ to be the injection which sends $\left(r_{1}, \ldots, r_{|A|-1}\right)$ to $\left(r_{1}, r_{2}, \ldots, r_{u-1}, 0, r_{u}, r_{u+1}, \ldots, r_{|A|-1}\right)$, and define $i_{2}: \Delta^{|A|-2}$ $\rightarrow \Delta^{|A|-1}$ to be the injection which sends $\left(r_{1}, \ldots, r_{|A|-1}\right)$ to $\left(r_{1}, r_{2}, \ldots, r_{u+l-2}, 0, r_{u+l-1}, \ldots, r_{|A|-1}\right)$. In other words, $i_{1}$ inserts a zero in the $u$ th coordinate so that $\zeta_{A} \circ i_{1}$ has a zero in the $(k+1)$ st coordinate, and similarly $\zeta_{A} \circ i_{2}$ has a zero in the $(k+l)$ th coordinate. Then as maps from $\Delta^{|\boldsymbol{A}|-2} \times T^{b}$ to $G$, where $b=b(A)=b(A-\{k+1\})=b(A-\{k+l\})$,

$$
\psi_{A-\{k+l\}} \circ(1 \times \pi)=\psi_{A} \circ\left(i_{1} \times 1\right) \text { and } \psi_{A-\{k+l\}}=\psi_{A} \circ\left(i_{2} \times 1\right) \text {. }
$$

17. Filter $G$ now by defining $G_{s}$ to be the closed set $\left\{\vec{z} \in G: z_{k} \neq 0\right.$ for at most $s$ values of $k\}$. Then $G_{0}=\varnothing$ and $G_{m}=G$. Also, if $0<s<m$, then by $\S 15$ (iii)

$$
G_{s}-G_{s-1}=\cup\left\{\psi_{A}\left(\operatorname{Int} \Delta^{s-1} \times T^{b(A)}\right):|A|=s\right\} .
$$

By $\$ 15$ (ii), this union is disjoint. Hence if $0<s<m$, then the integral homology $H_{*}\left(G_{s}, G_{s-1}\right)$ is a direct sum with one summand

$$
H_{*}\left(\psi_{A}\left(\Delta^{s-1} \times T^{b(A)}\right), \psi_{A}\left(\partial \Delta^{s-1} \times T^{b(A)}\right)\right)
$$

for each subset $A$ of $\{1,2, \ldots, m\}$ having $s$ elements. Note that by $\$ 15(\mathrm{i})$, the summand for $A$ is isomorphic via $\psi_{A^{*}}$ to $H_{*}\left(\Delta^{s-1} \times T^{b(A)}, \partial \Delta^{s-1} \times T^{b(A)}\right)$. Also, $H_{s-1+b(A)}\left(\Delta^{s-1} \times T^{b(A)}, \partial \Delta^{s-1} \times T^{b(A)}\right)$ is infinite cyclic. The manifold $\Delta^{s-1} \times$ $T^{b(A)}$ has a standard orientation. Let $\iota_{A}$ denote the image in $H_{s-1+b(A)}\left(G_{s}, G_{s-1}\right)$ under $\psi_{A^{*}}$ of the corresponding generator.

Define a proper subset $A$ of $\{1,2, \ldots, m\}$ to be thick if $A$ omits no consecutive pair of indices (consecutive in the cyclic sense, of course), that is, if $j \notin A$ implies $j+1 \in A$. Note that the pair $\left(\Delta^{s-1} \times T^{b(A)}, \partial \Delta^{s-1} \times T^{b(A)}\right)$ has homology in degree $m-1$ if and only if $A$ is thick, and has homology in degree $m-2$ only if $A$ omits at most one consecutive pair of indices.

Suppose $A$ has a long block beginning at $k+1$ and ending at $k+l$, and $|A|=s$. According to $\S 16$, the connecting homomorphism for the exact sequence 
of the triple $\left(G_{s}, G_{s-1}, G_{s-2}\right)$ maps $\iota_{A}$ to a sum in which the summand indexed by $A-\{k+1\}$ is $\pm a_{k+1} l_{A-\{k+1\}}$ and the summand indexed by $A-\{k+l\}$ is $\pm \iota_{A}-\{k+l\}$.

18. Consider now the spectral sequence of the filtration $\left\{G_{s}\right\}$ in which $E_{s, t}^{1}=$ $H_{s+t}\left(G_{s}, G_{s-1}\right)$ and $d_{s, t}^{1}: E_{s, t}^{1} \rightarrow E_{s-1, t}^{1}$ is the connecting homomorphism of the triple $\left(G_{s}, G_{s-1}, G_{s-2}\right)$. In total degree $s+t=m-1$,

$$
E_{s, m-1-s}^{1}=\bigoplus\left\{Z_{\iota_{A}}: A \text { is thick and }|A|=s\right\} .
$$

Note that if $s<m / 2$, then no thick $A$ of order $s$ exists, so $E_{s, m-1-s}^{1}=0$. If $m$ is even and $s=m / 2$, then there exist precisely two thick subsets with $s$ elements, but neither has any long blocks. Hence by a remark in $\$ 17$, for $r=$ $1,2,3, \ldots, d_{m / 2, m / 2-1}^{r}$ is the zero homomorphism.

Assume now that $m / 2<s<m$, and that $A$ has $s$ elements and is thick. Then $A$ has at least one long block. Choose such a block, say beginning at $k+1$. Define $A^{\prime}$ to be $(A \cup\{k\})-\{k+1\}$. Then $A^{\prime}$ has $s$ elements, is thick, and has a long block ending at $k$. Let $A^{\prime \prime}$ be derived from $A^{\prime}$ similarly by altering membership at the beginning of this new long block, and define $A^{(j)}=\left(A^{(j-1)}\right)^{\prime}$ inductively in the same way for all positive integers $j$. Note that $A^{(m-s)}=A+1=\{k+1: k \in A\}$. Therefore $A^{((m-s) m)}=A$. Suppose $d_{s, m-1-s}^{1}\left(\sum_{B} \lambda_{B} \iota_{B}\right)=0$, where $B$ runs over all thick subsets with $s$ elements, and the $\lambda_{B}$ 's are integers. In particular the coefficient of $\iota_{A-\{k+1\}}$ in the direct sum expansion of $d_{s, m-1-s}^{1} \Sigma_{B} \lambda_{B} \iota_{B}$ described in $\$ 17$ must be zero. This says $\pm a_{k+1} \cdot \lambda_{A} \pm \lambda_{A^{\prime}}=0$, i.e., $\lambda_{A^{\prime}}= \pm a_{k+1} \cdot \lambda_{A}$. Inductively, then, we see that $\lambda_{A}=\lambda_{A^{(m-s) m}}= \pm\left(a_{1} a_{2} \cdots a_{m}\right)^{m-s} \lambda_{A}$. Since we are assuming that $a_{1} a_{2} \cdots a_{m}>1$, it follows that $\lambda_{A}=0$. There was nothing special about $A$, however, so actually $d_{s, m-1-s}^{1}$ is a monomorphism. Therefore if $m / 2<s<m$, then nothing in total degree $m-1$ of filtration $s$ survives to $E_{s, m-1-s}^{2}$, let alone to $E^{\infty}$.

19. Suppose $\vec{z} \in G$ and for all $k, z_{k} \neq 0$, i.e., $\vec{z} \in G_{m}-G_{m-1}$. If we write $z_{k}=r_{k} \exp \left\{i \theta_{k}\right\}$, then $r_{1}^{2}+r_{2}^{2}+\cdots+r_{m}^{2}=1$ and $a_{k} \cdot \theta_{k}+\theta_{k+1} \equiv 0(\bmod 2 \pi Z)$ for all $k$.

Let $d=a_{1} a_{2} \cdots a_{m}+(-1)^{m-1}$, the determinant of the matrix of exponents for the monomials of $f$. The system of equations modulo $2 \pi Z$ for the $\theta_{k}$ 's has precisely $d$ solutions, in each of which each angle $\theta_{k}$ is an integral multiple of $2 \pi / d$. To see this, set $\theta_{2}=-a_{1} \cdot \theta_{1}, \theta_{3}=-a_{2} \cdot \theta_{2}, \ldots, \theta_{m}=-a_{m-1} \cdot \theta_{m-1}$ and then observe that $-a_{m} \cdot \theta_{m} \equiv \theta_{1}(\bmod 2 \pi Z)$ if and only if $(-1)^{m} a_{1} a_{2} \cdots a_{m} \theta_{1}=\theta_{1}+2 \pi j$ for some integer $j$.

Denote the simplex in $\overline{G-G_{m-1}}$ on which $\theta_{1} \equiv 2 \pi j / d$ by $\sigma_{j}$. Then $E_{m,-1}^{1}$, which is $H_{m-1}\left(G, G_{m-1}\right)$, is free on $d$ generators, namely the homology classes $\left[\sigma_{j}\right]$ of the relative cycles $\sigma_{j}, j=1,2, \ldots, d$.

Denote by $x_{j}$ the difference $\left[\sigma_{j}\right]-\left[\sigma_{j+1}\right], j=1,2, \ldots, m$.

LeMMA. $x_{j} \in E_{m,-1}^{2}$ and $x_{j}-x_{l} \in E_{m,-1}^{\infty}$ for any $j$ and $l$.

Proof. It is convenient for the purposes of this proof to use simplicial homology, regarding $G$ as a simplicial complex in which for each proper subset $A$ of 
$\{1,2, \ldots, m\}$ the image of $\psi_{A}$ is a subcomplex, and likewise each $\sigma_{j}$ is a subcomplex.

Fix $j$ and write $I=[0,1]$. Define for each $k=0,1,2, \ldots, m-1$ a map $\tau_{k}: \Delta^{m-2} \times I \rightarrow G_{m-1}$ as a composite $\tau_{k}=\psi_{\{1,2, \ldots, m\}-\{k\}}{ }^{\circ}(1 \times \alpha)$, in which $1 \times \alpha: \Delta^{m-2} \times I \rightarrow \Delta^{m-2} \times T^{1}=\Delta^{m-2} \times S^{1}$ and $\alpha: I \rightarrow S^{1}$ is defined by the formula $\alpha(t)=\exp \left\{(-1)^{k} a_{1} a_{2} \cdots a_{k}(j+t) \cdot 2 \pi i / d\right\}$. In other words, $\tau_{k}\left(r_{1}, r_{2}, \ldots, r_{m-1}, t\right)=\left(r_{1} w_{1}, \ldots, r_{k-1} w_{k-1}, 0, r_{k} w_{k+1}, \ldots, r_{m-1} w_{m}\right)$ where, for $l=0,1,2, \ldots, m-1$,

$$
w_{k+1+l}=\left(w_{k+l}\right)^{-a_{k+l}}=\exp \left\{(-1)^{k+l} a_{1} a_{2} \cdots a_{k+l}(j+t) \cdot 2 \pi i / d\right\} .
$$

Note that if $k+l \geqslant m$, since $(-1)^{m} a_{1} a_{2} \cdots a_{m}=1+(-1)^{m} d$, it follows that $w_{k+1+l}=w_{k+l-m+1}=\exp \left\{(-1)^{k+l-m} a_{1} a_{2} \cdots a_{k+l-m}\left(1+(-1)^{m} d\right) \cdot(j+t) \cdot 2 \pi i / d\right\}$.

If we impose on $\Delta^{m-2} \times I$ the simplicial structure obtained in the usual way by means of joins, orienting $I$ from 0 to 1 , then $\Delta^{m-2} \times I$ becomes a simplicial complex of dimension $m-1$. The sum of all the $(m-1)$-simplices is a chain in $C_{m-1}\left(\Delta^{m-2} \times I\right)$. Denote its image in $C_{m-1}\left(G_{m-1}\right)$ under $\tau_{k \sharp \text {, after sufficient }}$ subdivision and simplicial approximation on $\Delta^{m-2} \times \operatorname{Int} I$, by $\tau_{k}$ as well (a slight abuse of notation). Define a chain $\delta_{j}$ in $C_{m-1}\left(G_{m-1}\right)$ by $\delta_{j}=\sum_{k=0}^{m-1}(-1)^{k} \tau_{k}$. It is easy to see that $\partial\left(\sigma_{j}-\sigma_{j+1}+\delta_{j}\right) \in C_{m-2}\left(G_{m-2}\right)$. Since $\delta_{j}$ has filtration $m-1$, this proves that $\left[\sigma_{j}\right]-\left[\sigma_{j+1}\right] \in E_{m,-1}^{2}$.

To prove the second statement, we must be more precise about $\partial\left(\sigma_{j}-\sigma_{j+1}+\delta_{j}\right)$. If $k$ and $k^{\prime}$ are integers, write $k \ll k^{\prime}$ to mean $k+1<k^{\prime}$. For each pair $k$ and $k^{\prime}$ of indices such that $0 \leqslant k \ll k^{\prime} \leqslant m-1$, define maps $H_{k, k^{\prime}}^{(0)}$ and $H_{k, k^{\prime}}^{(1)}: \Delta^{m-3} \times I \times I$ $\rightarrow G_{m-2}$ as follows.

$$
\begin{aligned}
& H_{k, k^{\prime}}^{(0)}(\vec{r}, t, u)=\psi_{\{1,2, \ldots, m\}-\left\{k, k^{\prime}\right\}}\left(\vec{r}, \exp \left\{(-1)^{k} a_{1} a_{2} \cdots a_{k} \cdot u(j+t) \cdot 2 \pi i / d\right\},\right. \\
& \left.\exp \left\{(-1)^{k^{\prime}} a_{1} a_{2} \cdots a_{k^{\prime}} \cdot u(j+t) \cdot 2 \pi i / d\right\}\right) . \\
& H_{k, k^{\prime}}^{(1)}(\vec{r}, t, u)=\psi_{\{1,2, \ldots, m\}-\left\{k, k^{\prime}\right\}} \\
& \cdot\left(\vec{r}, \exp \left\{(-1)^{k} a_{1} a_{2} \cdots a_{k} \cdot\left[u(j+t)+t(-1)^{m} d\right] \cdot 2 \pi i / d\right\},\right. \\
& \left.\exp \left\{(-1)^{k^{\prime}} a_{1} a_{2} \cdots a_{k^{\prime}} \cdot u(j+t) \cdot 2 \pi i / d\right\}\right) \text {. }
\end{aligned}
$$

(If $k=0$ and $k^{\prime}=m-1$, then $b\left(\{1,2, \ldots, m\}-\left\{k, k^{\prime}\right\}\right)=1$ and we suppress the last argument of $\psi_{\{1,2, \ldots, m\}-\left\{k, k^{\prime}\right\}}$ in both maps.)

Impose the join simplicial structure on $\Delta^{m-3} \times I \times I$. Let $\beta_{1}$ in $C_{m-2}\left(\Delta^{m-3} \times I \times I\right)$ be the sum of all the $(m-2)$-simplices in the subcomplex $\Delta^{m-3} \times I \times\{1\}$. Then a straightforward computation shows that

$$
\partial\left(\sigma_{j}-\sigma_{j+1}+\delta_{j}\right)=\sum_{0<k \ll k^{\prime}<m-1}(-1)^{k+k^{\prime}-1}\left(\left(H_{k, k^{\prime}}^{(0)}\right)_{\sharp} \beta_{1}-\left(H_{k, k^{\prime}}^{(1)}\right)_{\sharp} \beta_{1}\right) .
$$

Now let $\gamma$ in $C_{m-1}\left(\Delta^{m-3} \times I \times I\right)$ be the sum of all the $(m-1)$-simplices, and let $\beta_{0}$ in $C_{m-2}\left(\Delta^{m-3} \times I \times I\right)$ be the sum of all the $(m-2)$-simplices in the 
subcomplex $\Delta^{m-3} \times I \times\{0\}$. A final boundary computation shows that

$$
\begin{aligned}
\partial\left\{\sigma_{j}-\sigma_{j+1}+\delta_{j}+\sum_{0<k \ll k^{\prime}<m-1}\left(\left(H_{k, k^{\prime}}^{(0)}\right)_{\sharp} \gamma-\left(H_{k, k}^{(1)}\right)_{\sharp} \gamma\right)\right\} \\
=\sum_{0<k \ll k^{\prime}<m-1}(-1)^{k+k^{\prime}-1}\left(\left(H_{k, k^{\prime}}^{(0)}\right)_{\sharp} \beta_{0}-\left(H_{k, k^{\prime}}^{(1)}\right)_{\sharp} \beta_{0}\right) .
\end{aligned}
$$

Note that the restrictions of $H_{k, k^{\prime}}^{(0)}$ and $H_{k, k^{\prime}}^{(1)}$ to $\Delta^{m-3} \times I \times\{0\}$ are independent of $j$. Hence modulo chains of filtration lower than $m,\left(\sigma_{j}-\sigma_{j+1}\right)-\left(\sigma_{l}-\sigma_{l+1}\right)$ is a permanent cycle. This proves the second statement of the lemma.

20. It will be useful to record part of the result of the last boundary calculation in $\S 19$ in the following form.

In $E_{m-2,0}^{2}$, which, according to $\$ 17$ is the quotient by the image $d_{m-1,0}^{1}\left(\bigoplus_{|A|=m-1} Z \iota_{A}\right)$ of a subgroup of

$$
\underset{|B|=m-2}{\bigoplus} H_{m-2}\left(\psi_{B}\left(\Delta^{m-3} \times T^{b(B)}\right), \psi_{B}\left(\partial \Delta^{m-3} \times T^{b(B)}\right)\right),
$$

$d_{m,-1}^{2} x_{j}$ is represented by a class whose component indexed by $B=\{1,2, \ldots, m-$ $2\}$ is $(-1)^{m} \iota_{\{1,2, \ldots, m-2\}}$, but whose component indexed by $B=\{0,1, \ldots, m-1\}$ $-\{k, k+1\}$ is zero for $k=0,1,2, \ldots, m-2$.

LEMMA. If for some integer $\lambda$ the multiple $\lambda x_{j}$ survives to $E_{m,-1}^{3}$, then $d \mid \lambda$.

Proof. Assume that $d_{m,-1}^{2} \lambda x_{j}=0$. Then some class in $E_{m-2,0}^{1}$ whose component indexed by $\{1,2, \ldots, m-2\}$ is $\left.(-1)^{m} \lambda_{\imath}, 2, \ldots, m-2\right\}$ and whose component indexed by $\{0,1,2, \ldots, m-1\}-\{k, k+1\}$ is zero for $k=0,1,2, \ldots, m-2$ actually belongs to the image of $d_{m-1,0}^{1}$; say it is $d^{1} \sum_{k=1}^{m} \lambda_{k} \imath_{\{1,2, \ldots, m\}-\{k\}}$. The rest of the argument is similar to the end of $\$ 18$. According to the result at the end of $\S 17$, refined to take careful account of signs, $\lambda_{k+1}=a_{k+1} \cdot \lambda_{k}$ for $k=1,2, \ldots, m$ -2 ,

$$
\lambda_{1}=(-1)^{m} a_{1} \cdot \lambda_{m} \text { and }(-1)^{m}\left(a_{m} \cdot \lambda_{m-1}-\lambda_{m}\right)=(-1)^{m} \lambda .
$$

Hence $\lambda=(-1)^{m} \lambda_{0} \cdot d$. This proves the lemma.

21. We can now compute $E_{s, t}^{\infty}$ when $s+t=m-1$. First recall that $E_{m,-1}^{1}$, which is $H_{m-1}\left(G, G_{m-1}\right)$, is free abelian of rank $d$ on the basis $\left[\sigma_{j}\right], j=1,2, \ldots, d$. The map $\left(z_{1}, z_{2}, \ldots, z_{m}\right) \mapsto\left(\left|z_{1}\right|,\left|z_{2}\right|, \ldots,\left|z_{m}\right|\right)$ sends the pair $\left(G, G_{m-1}\right)$ in $\mathbf{C}^{m}$ to the simplex pair $\left(\Delta^{m-1}, \partial \Delta^{m-1}\right)$ in $\mathbf{R}^{m}$. In fact, this map induces a morphism of the spectral sequence $\left\{E_{*, *}^{r}\right\}$ into the spectral sequence associated with the corresponding filtration (by the number of nonzero variables) on $\Delta^{m-1}$. Applying this morphism to $d^{1}: E_{m,-1}^{1} \rightarrow E_{m-1,-1}^{1}$, we see that no integral combination $\sum_{j=1}^{d} \lambda_{j}\left[\sigma_{j}\right]$ can survive to $E_{m,-1}^{2}$ unless $\sum_{j=1}^{d} \lambda_{j}=0$. According to the lemma of $\S 19$, however, $x_{j}=\left[\sigma_{j}\right]-\left[\sigma_{j+1}\right]$ does survive, and hence the survivors to $E_{m,-1}^{2}$ are precisely the integral span of the classes $x_{j}, j=1,2, \ldots, d-1$.

The weights $w_{1}, w_{2}, \ldots, w_{m}$ associated with the weighted homogeneous polynomial $f$ satisfy $a_{j} / w_{j}+1 / w_{j+1}=1$ for all $j$. Let

$$
\begin{aligned}
n_{j}= & (-1)^{m-1}+(-1)^{m-2} a_{j-1}+(-1)^{m-3} a_{j-2} a_{j-1}+\cdots \\
& +(-1)^{m-k} a_{j-k+1} a_{j-k+2} \cdots a_{j-1}+\cdots+a_{1} a_{2} \cdots a_{j-1} a_{j+1} \cdots a_{m} .
\end{aligned}
$$


Then it is easy to see that $1 / w_{j}=n_{j} / d$. According to Milnor and Orlik [4], the rank $\mu$ of $H_{m-1}\left(\overline{F_{0}}\right)$ is $\left(w_{1}-1\right)\left(w_{2}-1\right) \cdots\left(w_{m}-1\right)$. An easy computation shows that $\mu=a_{1} a_{2} \cdots a_{m}$. Hence $\mu=d-1$ if $m$ is odd and $\mu=d+1$ if $m$ is even.

According to $\S 18, E_{s, m-s-1}^{2}=0$ unless $s=m$ or $m$ is even and $s=m / 2$. In the latter case, $E_{m / 2, m / 2-1}^{r}$ has rank at most 2. Since, by the Deformation Lemma of $\$ 3$, $i_{*}: H_{m-1} G \rightarrow H_{m-1} F_{0}$ is an epimorphism, it follows that it is actually an isomorphism, that $E_{m,-1}^{\infty}$ has rank $d-1$, and that when $m$ is even $E_{m / 2, m / 2-1}^{\infty}$ has rank 2 .

LEMMA. $E_{m,-1}^{\infty}$ is free abelian, generated by the $d-1$ differences $x_{1}-x_{2}, x_{2}-$ $x_{3}, \ldots, x_{d-1}-x_{d}$.

Proof. Because of the rank considerations above, we need only establish that the given differences generate $E_{m,-1}^{\infty}$. We know that $E_{m,-1}^{2}$ is spanned by the $x_{j}$ 's. Suppose $\sum_{j=1}^{d} \lambda_{j} x_{j} \in E_{m,-1}^{\infty}$. Then, by the lemma of $\S 19,\left(\sum_{j=1}^{d} \lambda_{j}\right) x_{1} \in E_{m,-1}^{\infty}$. It follows then from the lemma of $\S 20$ that $d \mid \sum_{j=1}^{d} \lambda_{j}$. But $0=x_{1}+x_{2}+\cdots+x_{d}$, and so, after subtracting the appropriate multiple of this expression, we may assume that $\sum_{j=1}^{d} \lambda_{j}=0$. This proves the lemma.

22. It remains to compute the action of the characteristic map $h_{*}$ in $E_{*, *}^{\infty}$, using the formula

$$
h\left(z_{1}, z_{2}, \ldots, z_{m}\right)=\left(z_{1} \cdot \exp \left\{2 \pi i / w_{1}\right\}, z_{2} \cdot \exp \left\{2 \pi i / w_{2}\right\}, \ldots, z_{m} \cdot \exp \left\{2 \pi i / w_{m}\right\}\right) \text {. }
$$

In case $m$ is even, the two cells in filtration $m / 2$ which carry homology of total degree $m-1$ are actually spheres on which $h$ is homotopic to the identity map. Hence we will concentrate on $E_{m,-1}^{\infty}$.

From $1 / w_{j}=n_{j} / d$ we have $a_{j} n_{j}+n_{j+1}=d$ for all $j$, and it follows that the greatest common divisor $\operatorname{gcd}\left(n_{j}, d\right)$ is independent of $j$, say $\operatorname{gcd}\left(n_{j}, d\right)=q$ for all $j$. Since $h_{\sharp} \sigma_{j}=\sigma_{j+n_{1}}$, it follows that $h_{*}\left(x_{j}-x_{j+1}\right)=x_{j+n_{1}}-x_{j+n_{1}+1}$. Thus there is a basis for $E_{m,-1}^{\infty}$ in which the matrix for $h_{*}$ is obtained from the $d \times d$ block-diagonal matrix having $q$ nonzero blocks, each of them the $d / q \times d / q$ cyclic permutation matrix

$$
\left(\begin{array}{ccccc}
0 & 1 & 0 & \ldots & 0 \\
0 & 0 & 1 & \ldots & 0 \\
\vdots & \vdots & \vdots & & \vdots \\
0 & 0 & 0 & \cdots & 1 \\
1 & 0 & 0 & \cdots & 0
\end{array}\right)
$$

by deleting the last column and last two rows, then putting a row of (-1)'s at the bottom.

It is now easy to see that $\operatorname{ker}\left(I_{*}-h_{*}\right)$, which is isomorphic to $H_{m-1}(K)$, is free abelian of rank $q+(-1)^{m}$, while coker $\left(I_{*}-h_{*}\right)$, which is isomorphic to $H_{m-2}(K)$, is the direct sum of a free abelian group of rank $q+(-1)^{m}$ and a cyclic group of order $d / q$.

Let $H$ be the matrix for $h_{*}$ described above. Then it is also easy to see that $t I-H$ can be diagonalized by row and column operations as a matrix over the polynomial ring $Z[t]$ (even though $Z[t]$ is not a principal ideal domain); that is, 
there exist matrices $U(t)$ and $V(t)$ unimodular in the matrix ring of $Z[t]$ for which $U(t)[t I-H] V(t)$ is a diagonal matrix. This verifies for the polynomial $f$ a conjecture of Orlik [6, p. 265].

\section{REFERENCES}

1. V. I. Arnold, Normal forms of functions in neighborhoods of degenerate critical points, Russian Math. Surveys 29 (1974), 10-50.

2. Lawrence Graves, The theory of functions of real variables, 2nd ed., McGraw-Hill, New York, 1956.

3. John Milnor, Singular points of complex hypersurfaces, Ann. of Math. Studies, no. 61, Princeton Univ. Press, Princeton, N. J., 1963.

4. John Milnor and Peter Orlik, Isolated singularities defined by weighted homogeneous polynomials, Topology 9 (1970), 385-392.

5. Mutsuo Oka, On the homotopy types of hypersurfaces defined by weighted homogeneous polynomials, Topology 12 (1973), 19-32.

6. Peter Orlik, On the homology of weighted homogeneous manifolds, Proc. Second Conf. Transformation Groups. I, Lecture Notes in Math., vol. 298, Springer-Verlag, Berlin, 1972, pp. 260-269.

7. __ Singularities and group actions, Bull. Amer. Math. Soc. (N.S.) 1 (1979), 703-720.

8. Peter Orlik and Richard Randell, The monodromy of weighted homogeneous singularities, Invent. Math. 39 (1977), 199-211.

9. Peter Orlik and Philip Wagreich, Isolated singularities of algebraic surfaces with $C^{*}$-action, Ann. of Math. (2) 93 (1971), 205-228.

10. __ Algebraic surfaces with $k^{*}$-action, Acta. Math. 138 (1977), 43-81.

Department of Mathematics, Augsburg College, Minneapolis, Minnesota 55454 